\title{
Giant and high-risk gastrointestinal stromal tumor in the abdomino-pelvic cavity: A case report
}

\author{
YUJIAO WANG, JIE PENG and JINBAI HUANG \\ Department of Radiology, Clinical Medical College of Yangtze University, Jingzhou, Hubei 434025, P.R. China
}

Received February 1, 2015; Accepted December 14, 2015

DOI: $10.3892 / 01.2016 .4196$

\begin{abstract}
Gastrointestinal stromal tumors (GISTs) are benign mesenchymal tumors of the gastrointestinal tract. The clinical presentations of patients with GIST are variable and may be non-specific. The current study reports the case of a 66-year-old man that presented with a gradual enlargement of the abdomen, emaciation, hyperhidrosis and frequent and urgent micturition. A computed tomography (CT) scan of the abdomen revealed a large, heterogeneous, low density mass that occupied the entire abdomino-pelvic cavity. Magnetic resonance imaging (MRI) identified a high signal intensity on the T2 weighted image and an intermediate signal intensity on the T1 weighted image. A contrast enhanced CT scan and MRI demonstrated the uptake of contrast material. A biopsy revealed that the tumor was composed of spindle cells, and immunohistochemical analysis identified the presence of mast/stem cell growth factor receptors. Together, these results lead to a diagnosis of GIST. The clinical findings, imaging modalities and pathological studies suggested that the GIST was a large and high-risk tumor located in the abdomino-pelvic cavity. The final surgical results confirmed these findings. Following conservative treatment with imatnib (400 mg, daily) for 6 months, the tumor became smaller and was suitable for surgery, which the patient received in December 2014. The final surgery confirmed the high-risk GIST. Subsequent to the surgery, the patient was recommended to continue the use of imatnib with regular CT or MRI reexaminations every 3 months, which are planned to continue for 3 years.
\end{abstract}

\section{Introduction}

Gastrointestinal stromal tumor (GIST) is the most common mesenchymal neoplasm of the gastrointestinal tract and is defined by the expression of the mast/stem cell growth factor receptor, also termed cluster of differentiation (CD)117, a

Correspondence to: Mr. Jinbai Huang, Department of Radiology, Clinical Medical College of Yangtze University, 8 Hang Kong Road, Jingzhou, Hubei 434025, P.R. China

E-mail: jinbaihuang@126.com

Key words: giant, gastrointestinal stromal tumor, abdomino-pelvic tyrosine kinase growth factor receptor (1). The incidence of GIST is estimated to be 14-20 cases per million population. The most common site of GIST involvement is the stomach, followed by the small intestine (2). It is rarely located in extra-gastrointestinal sites, such as the omentum and mesentery. Tumors located in these regions are known as extra-gastrointestinal stromal tumors (3). The peak incidence of GIST is in adulthood (median age, 63 years) with no evident gender predilection (4). GIST may vary considerably in size and tumors $>10 \mathrm{~cm}$ in size are considered to be giant GISTs. GISTs $>5 \mathrm{~cm}$ in size with $\geq 10$ mitotic cells per 50 high-power fields (10/50 HPF) may become malignant and are considered to be high-risk tumors (5). The incidence of GIST is estimated to be $<20$ cases per million population (6). The standard treatment for GIST is surgery with a complete resection of the tumor. The prognosis is strictly associated with the size of the tumor and the completeness of the surgical resection.

\section{Case report}

A 66-year-old man that presented with metastatic pain in the right lower quadrant was admitted to the Clinical Medical College of Yangtze University (Jingzhou, Hubei, China) in February 2013. Ultrasound and computed tomography (CT) scans revealed a large mass in the lower abdominal and pelvic cavity. Histopathological examination suggested a diagnosis of spindle cell tumor. Following an initial improvement in the clinical symptoms, the patient discontinued the treatment regimen (imatnib, $400 \mathrm{mg} / \mathrm{d}$ ). Subsequent to discontinuation of medication, the patient experienced enlargement of the abdomen, emaciation, fever, hyperhidrosis and frequent and urgent micturition. The patient was re-admitted to hospital in May 2014. Clinical examination revealed that the abdomen was soft and enlarged with superficial abdominal varicose veins. A large mass was palpated that covered the entire abdomen. The liver and spleen were not palpable. A CT scan revealed the presence of a large soft tissue mass, which occupied almost the entire abdomino-pelvic cavity (Fig. 1), and measured $34 \times 19.1 \times 28.6 \mathrm{~cm}$ in size. The intestines were observed to be gathered at the back of the abdominal cavity, and a large segment of the bowel was embedded inside the mass. The mass was heterogeneous and possessed a slightly lower density than the liver. Certain strip-like lower density areas were also observed within the mass. The boundary of the mass was defined. A contrast enhanced CT scan demonstrated that the 


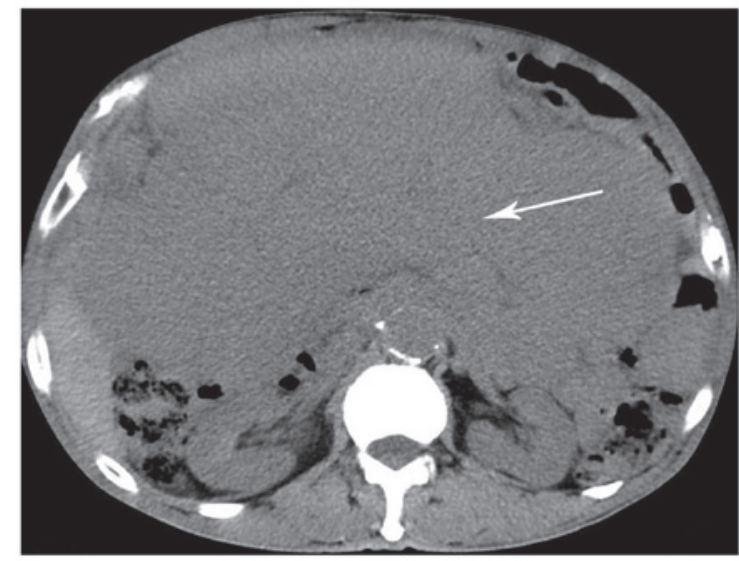

Figure 1. Computed tomography revealed the presence of a large soft tissue mass, which occupied the abdominal-pelvic cavity (arrow). The density of the mass was heterogeneous and slightly lower than the density of the liver. Certain strip-like lower density images were observed in the mass. The mass boundary was defined. The intestines were gathered at the back of the abdominal cavity. A large segment of the bowel was embedded in the mass.

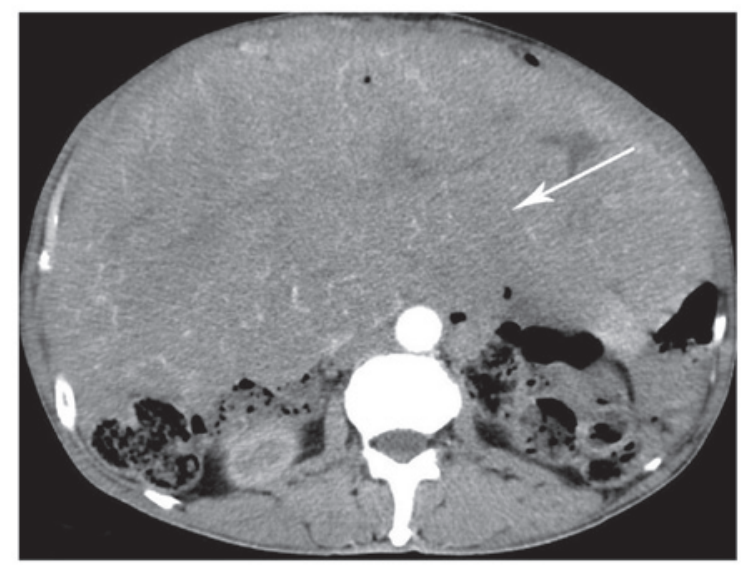

Figure 2. Contrast-enhanced computed tomography revealed that the mass was slightly enhanced. Numerous serpiginous small vessels were observed throughout the mass (arrow).

mass was slightly enhanced, with numerous serpiginous small vessels within the mass (Fig. 2). A computed tomography angiography scan revealed that the mass was supplied by the mesenteric artery (Fig. 3). A magnetic resonance imaging (MRI) scan revealed that the tumor possessed an equal signal on the $\mathrm{T} 1$ weighted image and a high signal on the $\mathrm{T} 2$ weighted image (Figs. 4 and 5). The enhancement pattern of the mass was similar between the MRI and CT scans (Fig. 6).

The CT and MRI results lead to a diagnosis of giant abdomino-pelvic hypervascular tumor. Following a biopsy, the diagnosis was altered to abdominal spindle cell tumor. Immunohistochemical tests were performed, the results of which were as follows: Expression of CD117, discovered on GIST-1, smooth muscle actin, human melanoma black 45; partial expression of protein S-100; no expression of cluster of differentiation-34, pancytokeratin, melanoma antigen and C-reactive protein; and a Ki67 index of 5-10\% (Figs. 7 and 8). A mitosis count of $<5 / 50$ HPF was observed. Overall, the results from the clinical and immunohistochemical tests lead to a final diagnosis of high-risk GIST. The final surgical

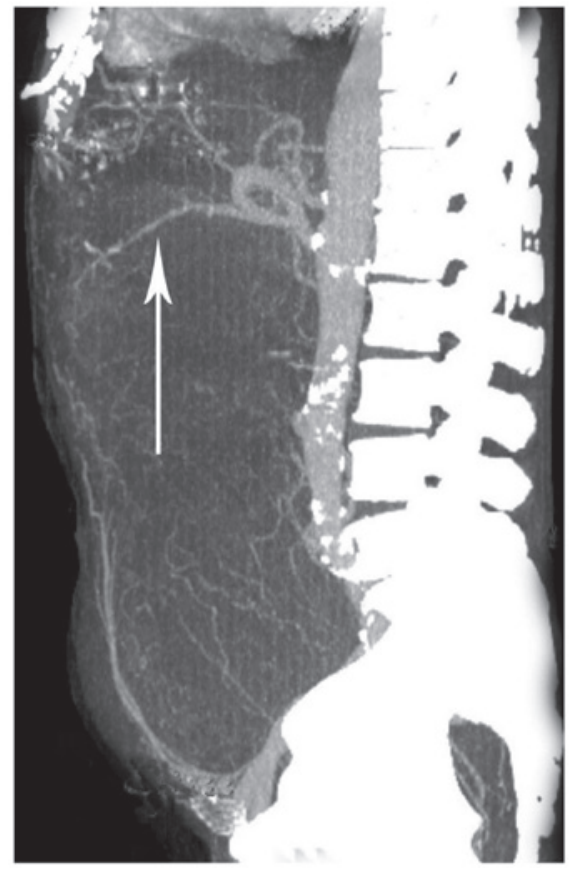

Figure 3. Computed tomography angiography demonstrated that the mass was supplied by the mesenteric artery (arrow), in which certain small vessels were observed.

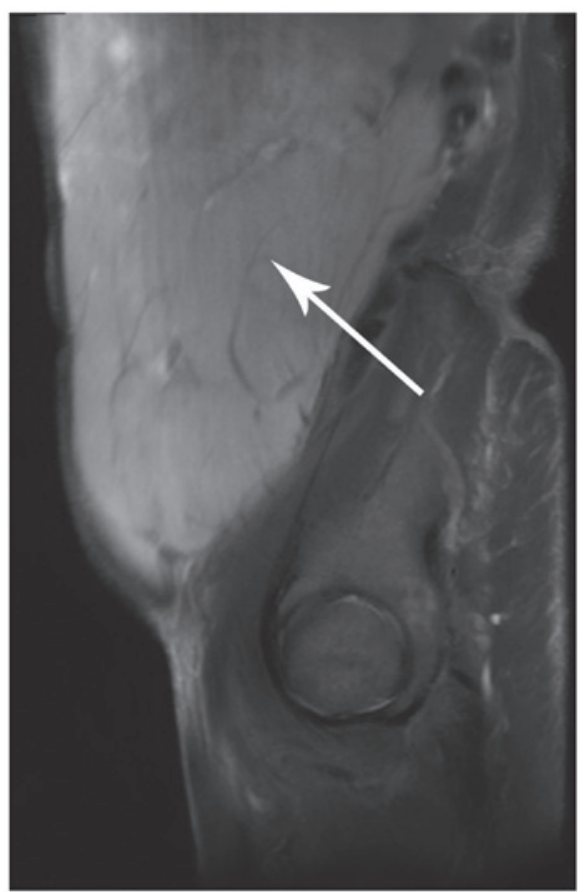

Figure 4. Sagittal T2-weighted magnestic resonance imaging revealed a homogeneous mass with a high signal intensity (arrow).

results confirmed these findings. Following conservative treatment with imatnib (400 mg, daily) for 6 months, the tumor became smaller and was suitable for surgery, which the patient received in December 2014. The final surgery confirmed the high-risk GIST. Subsequent to the surgery, the patient was recommended to continue the use of imatnib and receives regular CT or MRI reexaminations every 3 months, which are planned to continue for 3 years. 


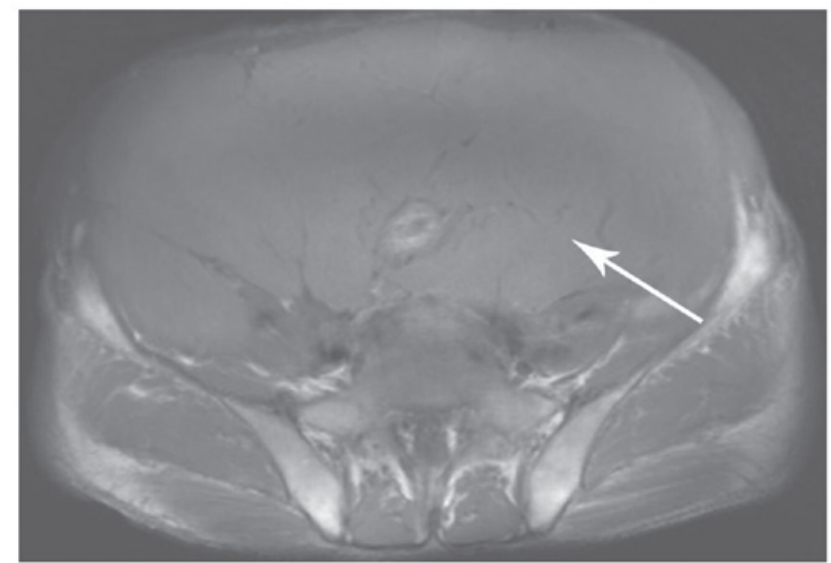

Figure 5. Axial T1-weighted magnetic resonance imaging revealed a homogeneous mass with an intemediate signal intensity (arrow).

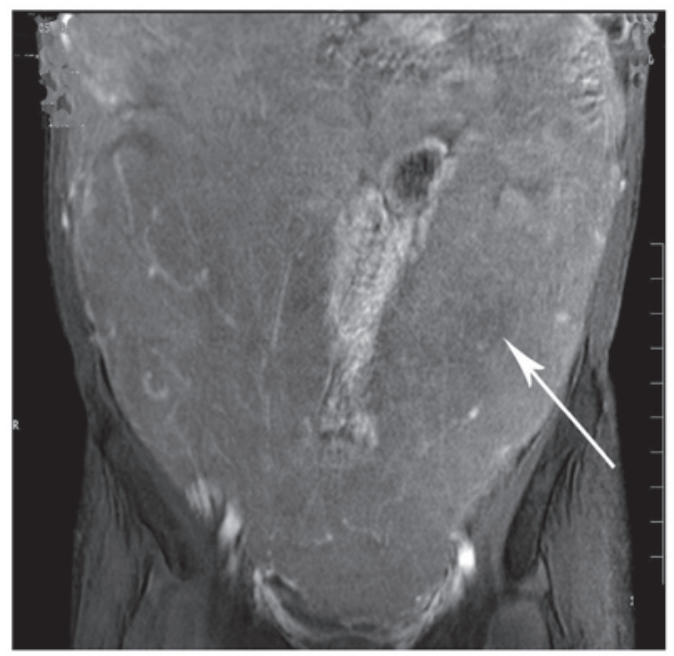

Figure 6. The enhancement pattern of the mass was similar between magnestic resonance imaging and computed tomography scans (arrow).

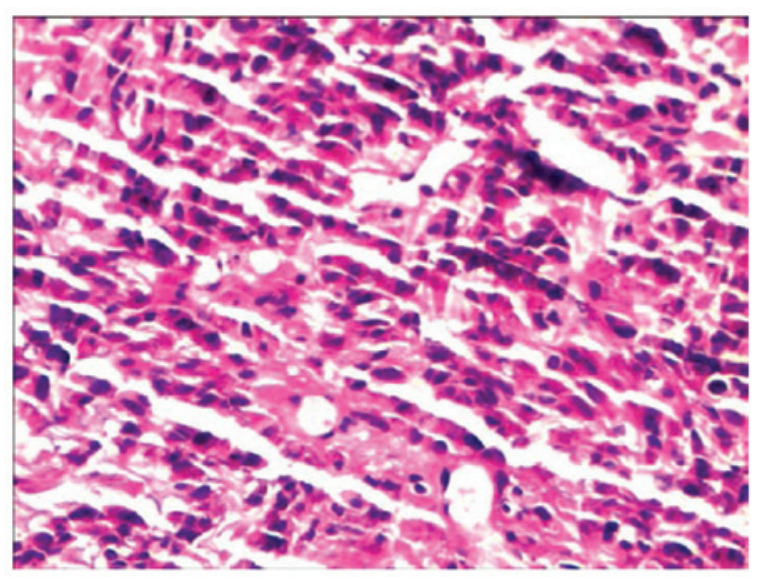

Figure 7. Hematoxylin and eosin staining revealed that the tumor was composed of spindle cells. Magnification, x400.

\section{Discussion}

GISTs are rare mesenchymal tumors that arise predominantly

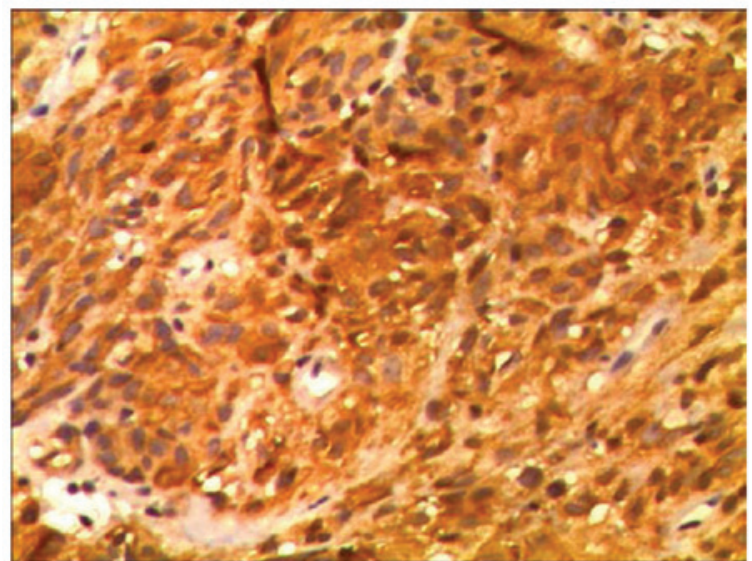

Figure 8. Immunohistochemical staining revealed that the tumor markedly expressed cluster of differentiation 117. Magnification, $\mathrm{x} 400$.

in the gastrointestinal tract, and may occur at any location between the esophagus and the rectum (7). The most common regions of the gastrointestinal tract affected are the stomach $(60 \%)$ and small intestine $(30 \%)(8,9)$. In total, $>90 \%$ of GISTs occur in adults $>40$ years old (median age, 63 years); however, GISTs have been identified in all age groups, including children (10). The majority of GISTs remain asymptomatic until they have reached a large size (11). Symptoms vary according to location and size of the tumor, and patients generally present with non-specific symptoms, such as abdominal pain, fatigue, dyspepsia, nausea, anorexia, weight loss, fever and obstruction of the intestines (12). GIST may be classified into five categories, according to the growth pattern of the tumor. The categories consist of the cross-wall, internal-wall, external-wall, intracavity and extra-gastrointestinal tract types (13). GISTs range in size of diameter between a few millimeters and $>30 \mathrm{~cm}$ (14). When the size of the mass measures $>10 \mathrm{~cm}$, the GIST is referred to as giant GIST $(15,16)$. The risk of malignancy of GISTs varies between very low and high based on the mitotic rate and size; GISTs $>5 \mathrm{~cm}$ with a mitotic rate of $>5 / 50 \mathrm{HPF}$ are considered to be high-risk tumors (8). The risk of malignancy is calculated according to the modified National Institutes of Health criteria (17), which classifies GIST tumors into 4 categories: Extremely low, low, intermediate and high-risk (18).

Multislice spiral computer tomography (MCST) is considered to be the optimal imaging method for the detection of GISTs (19). On CT and MRI, GISTs appear as large heterogeneous masses with areas of low attenuation from hemorrhage, necrosis or cyst formation. Severe hemorrhage and calcification within the mass is occasionally revealed, but lymphadenopathy is not a common finding. A malignant stromal tumor is observed on a CT scan as a mass $>5 \mathrm{~cm}$, possessing a lobulated contour and heterogeneity, with central necrosis or liquefaction, mesenteric infiltration, regional lymphadenopathy and exophytic growth. GISTs demonstrate conspicuous uniform or non-uniform enhancement on a contrast-enhanced scan. GISTs may be composed of thin, elongated spindle cells or plumper epithelioid cells. The most useful diagnostic marker for GIST is CD117, a cell-surface transmembrane tyrosine kinase, also known as c-Kit, which is immunohistochemically detectable in 95\% of GISTs (20). 
The current patient presented with characteristic features of GIST, which is demonstrated by the clinical features of the patient, CT and MRI imaging, biopsy findings and immunohistochemical staining. The mass was large and occupied almost the entire abdominal and pelvic cavity. No association between the mass and the adjacent gastrointestinal structure was observed on the CT scan. As the mass was large and did not exhibit any symptoms of intestinal obstruction, it was concluded that the mass was extra-gastrointestinal in origin. In addition, the mass was a hypervascular tumor, as certain tortuous enhanced vessels were observed within the mass in a contrast-enhanced CT scan. However, the tumor parenchyma did not clearly enhance in every stage of the contrast enhanced scan. Therefore, whether this inconsistent appearance is a characteristic feature of GIST requires clarification by additional studies of patients with similar characterisitcs to the present patient.

\section{References}

1. Novelli M, Rossi S, Rodriguez-Justo M, Taniere P, Seddon B, Toffolatti L, Sartor C, Hogendoorn PC, Sciot R, Van Glabbeke M, et al: DOG1 and CD117 are the antibodies of choice in the diagnosis of gastrointestinal stromal tumours. Histopathology 57: 259-270, 2010.

2. Su YY, Chiang NJ, Wu CC and Chen LT: Primary gastrointestinal stromal tumor of the liver in an anorectal melanoma survivor: A case report. Oncol Lett 10: 2366-2370, 2015.

3. Bár T, Sankot J, Adamová Z and Mičulka P: Extraintestinal GIST - case report. Rozhl Chir 94: 383-386, 2015 (In Czech).

4. Suryawanshi KH, Patil TB, Damle RP, Dravid NV and Surana A: Gastrointestinal stromal tumour of small intestine presenting as a mesenteric mass. J Clin Diagn Res 8: FD14-FD16, 2014.

5. Sashidharan P, Matele A, Matele U, Al Felahi N and Kassem KF: Gastrointestinal stromal tumors: A case report. Oman Med J 29: 138-141, 2014.

6. Liang X, Yu H, Zhu LH, Wang XF and Cai XJ: Gastrointestinal stromal tumors of the duodenum: Surgical management and survival results. World J Gastroenterol 19: 6000-6010, 2013.

7. Salari M, Ahadi M, Hoseini SM, Mokhtari E, Gafarzadehgan K, Hashemian HR, Esmaeili B and Vossoughinia $\mathrm{H}$ : Gastrointestinal Stromal Tumors in Northeastern Iran: 46 Cases During 2003-2012. Middle East J Dig Dis 7: 161-165, 2015.
8. Rammohan A, Sathyanesan J, Rajendran K, Pitchaimuthu A, Perumal SK, Srinivasan U, Ramasamy R, Palaniappan R and Govindan M: A gist of gastrointestinal stromal tumors: A review. World J Gastrointest Oncol 5: 102-112, 2013.

9. Antonopoulos P, Leonardou P, Barbagiannis N, Alexiou $\mathrm{K}$, Demonakou $\mathrm{M}$ and Economou N: Gastrointestinal and extragastrointestinal stromal tumors: Report of two cases and review of the literature. Case Rep Gastroenterol 8: 61-66, 2014.

10. Stamatakos M, Douzinas E, Stefanaki C, Safioleas P, Polyzou E, Levidou G and Safioleas M: Gastrointestinal stromal tumor. World J Surg Oncol 7: 61-70, 2009.

11. Sorour MA, Kassem MI, Ghazal A-H, El-Riwini MT and Abu Nasr A: Gastrointestinal stromal tumors (GIST) related emergencies. Int J Surg 12: 269-280, 2014.

12. Zhao X and Yue C: Gastrointestinal stromal tumor. J Gastrointest Oncol 3: 189-208, 2012.

13. Beham AW, Schaefer I-M, Schüler P, Cameron S and Ghadimi BM: Gastrointestinal stromal tumors. Int J Colorectal Dis 27: 689-700, 2012

14. Giuliani J and Bonetti A: The occurrence of gastrointestinal stromal tumors and second malignancies. J Gastrointest Cancer 46: 408-412, 2015.

15. Gerrish ST and Smith JW: Gastrointestinal stromal tumors - diagnosis and management: A brief review. Ochsner J 8: 197-204, 2008.

16. Dickhoff C, Leguit RJ, Slors JF, Vervenne WL and Bemelman WA: Giant rectal gastrointestinal stromal tumors: A report of two cases. Case Rep Gastroenterol 2: 54-69, 2008.

17. de Oliveira RP, Portari Filho PE, Iglesias AC, de Oliveira CA and Pannain VL: Comparative study of the different degrees of risk of gastrointestinal stromal tumor. Rev Col Bras Cir 42: 32-36, 2015.

18. Zhao WY, Xu J, Wang M, Zhang ZZ, Tu L, Wang CJ, Cao H and Zhang ZG: Evaluation of high-risk clinicopathological indicators in gastrointestinal stromal tumors for prognosis and imatinib treatment outcome. BMC Gastroenterol 14: 105-113, 2014.

19. Cai PQ, Lv XF, Tian L, Luo ZP, Mitteer RA Jr, Fan Y and Wu YP: CT characterization of duodenal gastrointestinal stromal tumors. AJR Am J Roentgenol 204: 988-993, 2015.

20. Serrano C and George S: Recent advances in the treatment of gastrointestinal stromal tumors. Ther Adv Med Oncol 6: 115-127, 2014. 